$01 ; 14 ; 15$

\title{
Аппаратная реализация нейроподобного генератора с импульсной и пачечной динамикой на основе системы фазовой синхронизации
}

\author{
(C) М.А. Мищенко, Д.И. Большаков, В.В. Матросов
}

Национальный исследовательский Нижегородский государственный университет им. Н.И. Лобачевского, Нижний Новгород

E-mail: mischenko@neuro.nnov.ru

Поступило в Редакцию 8 февраля 2017 г.

Предложена аппаратная модель нейрона на основе системы фазовой синхронизации с полосовым фильтром в цепи управления. Описаны основные конструктивные элементы системы. Экспериментально продемонстрировано существование различных динамических режимов, качественно похожих на импульсную и пачечную динамику нейронов.

DOI: 10.21883/PJTF.2017.13.44806.16737

Задача аппаратной реализации нейронов и их сетей возникла в конце 80-х годов XX века [1,2]. К этому времени было известно достаточно много математических моделей, описывающих динамику нейронов с различной степенью детализации [3-6]. Однако слабое развитие вычислительных технологий не позволяло проводить численные эксперименты даже с небольшими ансамблями модельных элементов. Огромным преимуществом на этом фоне обладали аппаратные или „кремниевые“ нейроны, поскольку скорость их работы определялась только конкретной схемотехнической реализацией и не ограничивалась количеством элементов, включенных в сеть. Было разработано множество различных схемотехнических реализаций нейронов, отличающихся по степени детализации и биологической правдоподобности $[1,7-10]$, по количеству воспроизводимых динамических режимов [11-14]. Также исследовалась и коллективная динамика аппаратных нейронных моделей [15-21]. 
В работе [22] авторами предложена математическая модель нейроподобного генератора на основе системы фазовой синхронизации с полосовым фильтром. Эта модель подробно исследована в работах [23,24]. Показано, что в такой системе при всех значениях параметров реализуется режим биений. При этом изменение напряжения на выходе полосового фильтра качественно похоже на динамику мембранного потенциала нейрона.

Преимуществом таких систем является их повсеместная распространенность в современной электронике и отработанные технологии промышленного изготовления. Использование систем фазовой автоподстройки для моделирования динамики нейронов рассматривалось в работах $[25,26]$.

В данной работе представлена аппаратная реализация модели нейрона на основе системы фазовой синхронизации с полосовым фильтром.

Математическая модель нейроподобного генератора имеет вид

$$
\begin{aligned}
& \dot{\varphi}=y, \\
& \dot{y}=z, \\
& \varepsilon_{1} \varepsilon_{2} \dot{z}=\gamma-\left(\varepsilon_{1}+\varepsilon_{2}\right) z-\left(1+\varepsilon_{1} \cos \varphi\right) y,
\end{aligned}
$$

где $\varphi-$ текущая разность фаз подстраиваемого и опорного генераторов, $\gamma$-начальная частотная расстройка, $\varepsilon_{1}, \varepsilon_{2}$ - параметры инерционности фильтра. Применительно к динамике нейрона переменную $y$ можно интерпретировать как описывающую изменение мембранного потенциала, параметры $\varepsilon_{1}$ и $\varepsilon_{2}$ позволяют задавать необходимый динамический режим, а $\gamma$ оказывает воздействие, сходное с воздействием внешнего тока в модели Ходжкина-Хаксли.

Анализ модели (1) показал, что в фазовом пространстве существуют аттракторы вращательного типа (переменная $\varphi$ неограниченно возрастает) различной сложности. Данным аттракторам соответствуют различные динамические режимы модели. Регулярным предельным циклам различной кратности соответствуют периодическая импульсная или пачечная (бёрстовая) активность. Для регулярных движений количество импульсов в пачке соответствует кратности предельного цикла. В случае, когда вместо предельного цикла в фазовом пространстве существует хаотический аттрактор, наблюдается пачечная динамика со случайным количеством импульсов в пачке. В работах $[23,24]$

Письма в ЖТФ, 2017, том 43, вып. 13 


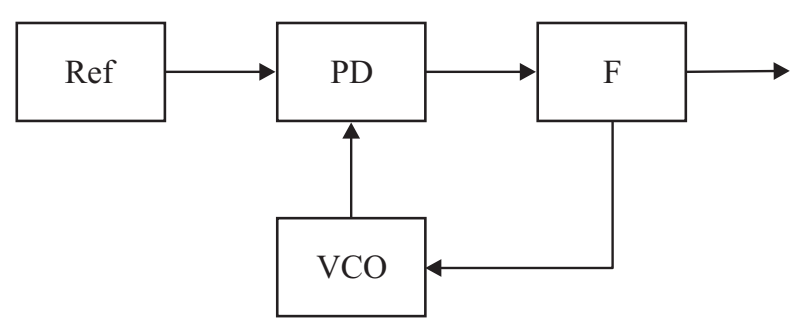

Рис. 1. Структурная схема аппаратной модели нейроподобного генератора на основе системы фазовой синхронизации. VCO - генератор, управляемый напряжением; Ref - опорный генератор; PD - фазовый дискриминатор; F фильтр в цепи управления.

представлено разбиение пространства параметров модели на области существования различных динамических режимов и изучены бифуркационные механизмы переходов между ними.

Аппаратная реализация разработана на основе классической структурной схемы системы фазовой синхронизации $[27,28]$ путем включения в цепь управления полосового фильтра вместо традиционно применяемого фильтра нижних частот (рис. 1).

Система фазовой автоподстройки частоты представляет собой генератор, управляемый напряжением (ГУН, VCO), охваченный петлей отрицательной обратной связи через фазовый дискриминатор и фильтр в цепи управления. Тип фильтра влияет на динамические и функциональные характеристики системы. В качестве опорного генератора использовался генератор прямоугольных импульсов с частотой $16 \mathrm{MHz}$.

В качестве основного элемента аппаратной реализации выбрана интегральная микросхема CD4046B фирмы Texas Instruments [29], содержащая в составе два фазовых компаратора, имеющих общий вход. Для аппаратной реализации модели (1) использовался фазовый компаратор, работающий по логической схеме „Исключающее ИЛИ“. Он имеет линейную зависимость среднего значения выходного напряжения от фазовой разности и всегда генерирует выходные колебания, которые в дальнейшем проходят через фильтр, входящий в состав цепи управления, сглаживающий логический выходной сигнал полной амплитуды. Следовательно, в контуре всегда присутствуют некоторая остаточная пульсация и некоторые фазовые изменения. Собственная частота ГУН изменялась в диапазоне 5-10 MHz. 


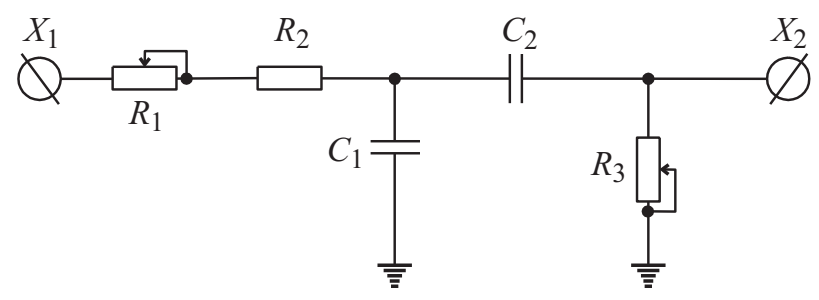

Рис. 2. Схема полосового фильтра в цепи управления.

Для управления частотой выходных колебаний и согласования блоков системы по частоте опорный генератор и ГУН подключены через делители частоты с изменяемым коэффициентом деления в диапазоне от 1 до 32000 .

Для воспроизведения динамики модели (1) использовался пассивный полосовой фильтр, состоящий из последовательно соединенных фильтров нижних и верхних частот. Схема полосового фильтра представлена на рис. 2, где $X_{1}-$ вход фильтра, на который поступает сигнал с фазового детектора, и $X_{2}$ - выход фильтра, сигнал с которого управляет частотой ГУН, а также является выходом модели нейроподобного генератора. Переменные резисторы $R_{1}$ и $R_{3}$ предусмотрены для перестройки фильтра, что соответствует изменению параметров $\varepsilon_{1}$, $\varepsilon_{2}$ модели (1). Диапазон изменения сопротивления резисторов $R_{1}$ и $R_{3}$ составляет от $100 \Omega$ до $5 \mathrm{k} \Omega$, остальные номиналы элементов фиксированы $R_{2}=2 \mathrm{k} \Omega, C_{1}=C_{2}=0.4 \mu \mathrm{F}$.

Для пересчета размерных параметров схемы в безразмерные параметры модели (1) использовались формулы

$$
\begin{aligned}
& \varepsilon_{1}=\frac{\Omega_{y}}{n}\left(R_{1}+R_{2}\right) C_{1}, \\
& \varepsilon_{2}=\frac{\Omega_{y}}{n} R_{3} C_{2}, \\
& \gamma=\frac{n}{\Omega_{y}}\left(\frac{\omega_{r e f}}{m}-\frac{\omega_{0}}{n}\right),
\end{aligned}
$$

где $\Omega_{y}=S E-$ полоса удержания ФАПЧ $(S-$ чувствительность ГУН, $E$ - максимальная возможная амплитуда сигнала на выходе фазового

Письма в ЖТФ, 2017, том 43, вып. 13 

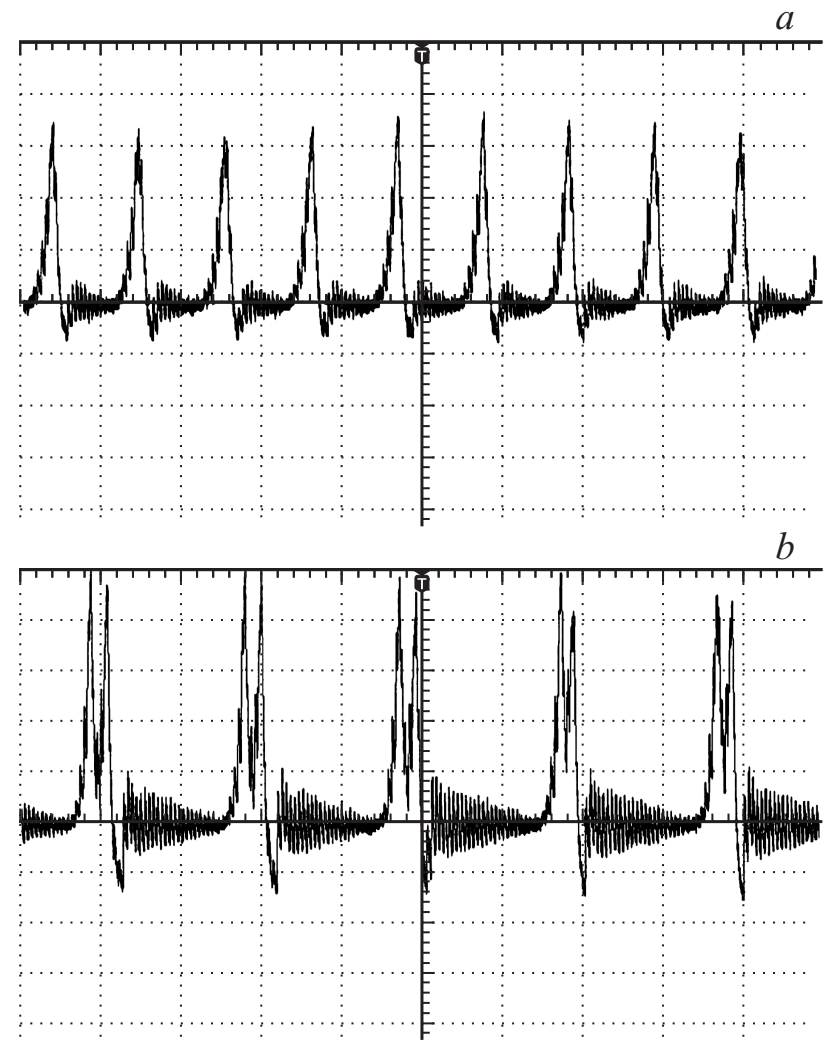

Рис. 3. Экспериментально записанные осциллограммы динамических режимов аппаратной модели нейроподобного генератора в случае регулярной импульсной активности $(a)$, регулярной пачечной активности с двумя $(b)$ и пятью $(c)$ импульсами в пачке, хаотической пачечной активности $(d)$.

детектора), $n$ и $m$ - коэффициенты деления частоты ГУН и опорного генератора соответственно, $\omega_{\text {ref }}=16 \mathrm{MHz}$ - частота опорного генератора, $\omega_{0}$ - частота свободных колебаний ГУН.

Разработанная схема реализована в виде аналогового электронного устройства. Путем подбора параметров схемы (сопротивления $R_{1}$ и $R_{3}$ и коэффициенты деления частоты $n$ и $m$ при фиксированной $\omega_{0}=5 \mathrm{MHz}$ )

Письма в ЖТФ, 2017, том 43, вып. 13 


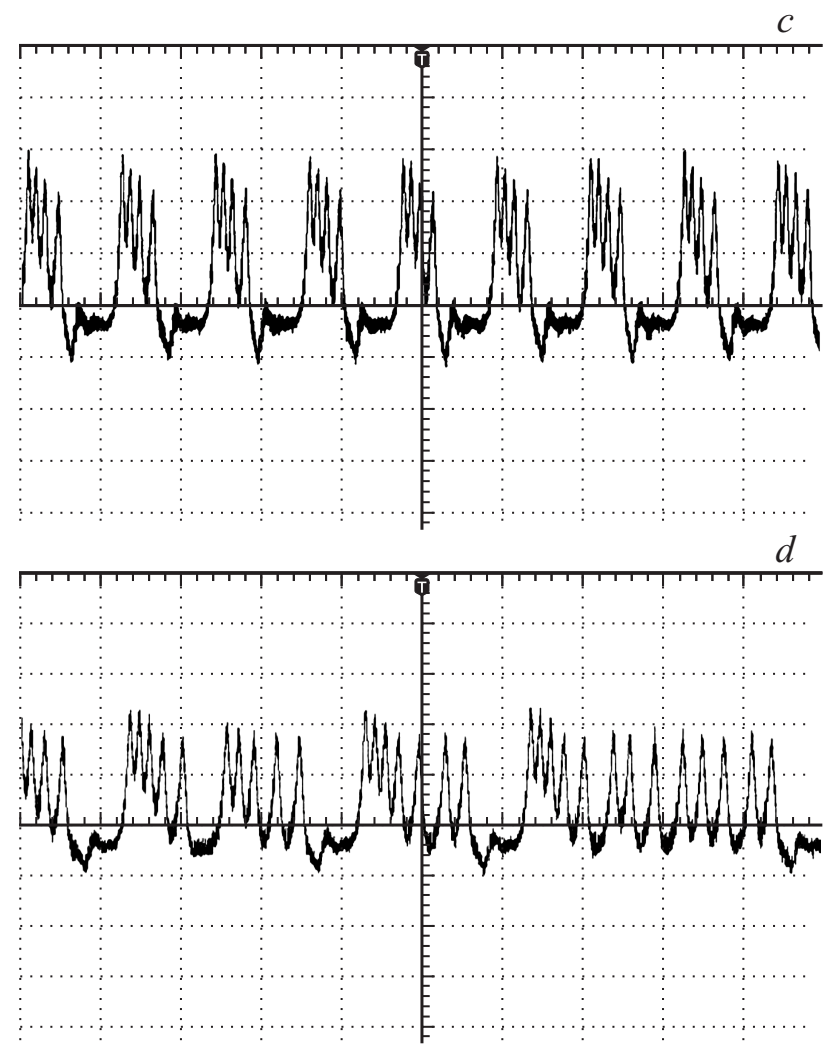

Рис. 3 (продолжение).

с учетом формул (2) и результатов теоретического анализа [24] обнаружены различные динамические режимы. Осциллограммы различных режимов, записанные при помощи осциллографа с выхода полосового фильтра в цепи управления, представлены на рис. 3.

В данной работе предложена аппаратная реализация модели нейроподобного генератора на основе системы фазовой синхронизации, ранее предложенной и исследованной теоретически в работах [22-24]. Экспериментально подтверждено существование периодической импульсной и пачечной (бёрстовой) активности, а также хаотической пачечной активности со случайным количеством импульсов в пачке.

Письма в ЖТФ, 2017, том 43, вып. 13 
Подобные динамические режимы качественно похожи на активность живых нейронов [30,31].

Разработанная аппаратная реализация нейроподобного генератора позволяет управлять частотой, амплитудой колебаний и выбирать необходимый динамический режим путем изменения всего трех параметров - величин сопротивлений $R_{1}$ и $R_{3}$ и коэффициента деления частоты $m$. Используемая в аппаратной реализации микросхема CD4046B достаточно распространена и доступна, поскольку применяется во множестве электронных устройств общего назначения. Этот факт позволяет говорить о доступности предлагаемой аппаратной модели нейрона и возможности ее миниатюризации за счет использования чиповой реализации микросхемы CD4046B. На разработанную схему нейроподобного генератора получен патент РФ на полезную модель [32].

Актуальность разработки аппаратных моделей нейронов обусловлена, во-первых, возможностью электронных устройств работать с большими ансамблями в режиме реального времени. Как любая реальная система, включая живые нейроны, аппаратная модель содержит случайные неоднородности в параметрах и внутренние источники шума, позволяя оценивать устойчивость систем и динамических режимов. Вовторых, электронный нейрон может быть базовой единицей параллельных электронных архитектур, более подходящих для моделирования крупномасштабных сетей, чем традиционные численные симуляции [33]. В-третьих, разработка аппаратных нейронов представляет интерес для такой перспективной области технологий, как нейропротезирование - создание интерфейсов между живыми нейронными сетями мозга и электронными нейроподобными устройствами для замещения функций поврежденных участков нейронных сетей мозга [34,35].

Исследование выполнено при финансовой поддержке РФФИ в рамках научного проекта № 16-31-60080 мол_а_дк (М.А. Мищенко) и № 16-32-00643 мол_а (М.А. Мищенко и Д.И. Большаков). Авторы благодарят за частичную поддержку Министерство образования и науки России в рамках соглашения № $3.820 .2014 /$ К (В.В. Матросов).

\section{Список литературы}

[1] Mead C. Analog VLSI and Neural Systems. Boston: Addison-Wesley, 1989.

[2] Mahowald M., Douglas R. // Nature. 1991. V. 354. N 6354. P. 515. 
[3] Hodgkin A.L., Huxley A.F. // J. Physiol. Blackwell Publish. 1952. V. 117. N 4. P. 500.

[4] FitzHugh R. // Biophys. J. 1961. V. 1. N 6. P. 445.

[5] Hindmarsh J.L., Rose R.M. // Proc. R. Soc. B. Biol. Sci. 1984. V. 221. N 1222. P. 87.

[6] Morris C., Lecar H. // Biophys. J. 1981. V. 35. N 1. P. 193.

[7] Indiveri G., Douglas R., Smith L. // Scholarpedia. 2008. V. 3. N 3. P. 1887.

[8] Indiveri G., Linares-Barranco B., Hamilton T.J. et al. // Front. Neurosci. 2011. V. 5. P. 73.

[9] Simoni M.F., Cymbalyuk G.S., Sorensen M.E. et al. // IEEE Trans. Biomed. Eng. 2004. V. 51. N 2. P. 342.

[10] Nishigami T., Torikai H. // Neural Networks. 2009. V. 22. N 5-6. P. 664.

[11] Wijekoon J.H.B., Dudek P. // Neural Networks. 2008. V. 21. N 2-3. P. 524.

[12] Savino G.V., Formigli C.M. // Biosystems. 2009. V. 97. N 1. P. 9.

[13] van Schaik A. // Neural Networks. 2001. V. 14. N 6-7. P. 617.

[14] Kohno T., Aihara K. // Neurocomputing. 2008. V. 71. N 7-9. P. 1619.

[15] Arthur J.V., Boahen K. // IEEE Trans. Neural Networks. 2007. V. 18. N 6. P. 1815.

[16] Poon C.-S., Zhou K. // Front. Neurosci. 2011. V. 5. P. 108.

[17] Kohno T., Sekikawa M., Li J. et al. // Front. Neurosci. 2016. V. 10. P. 273.

[18] Li J., Katori Y., Kohno T. // Front. Neurosci. 2012. V. 6. P. 183.

[19] Merolla P.A., Arthur J.V., Alvarez-Icaza R. et al. // Science. 2014. V. 345. N 6197. P. 668.

[20] Aggarwal A. // Neural Networks. 2016. V. 77. P. 29.

[21] Misra J., Saha I.A. // Neurocomputing. 2010. V. 74. N 1-3. Р. 239.

[22] Мищенко М.A. // Вестник Нижегородского университета им. Н.И. Лобачевского. 2011. Т. 5 (3). С. 279.

[23] Мищенко М.А., Шалфеев В.Д., Матросов В.В. // Известия вузов. Прикладная нелинейная динамика. 2012. Т. 20. № 4. С. 122.

[24] Matrosov V.V, Mishchenko M.A., Shalfeev V.D. // Eur. Phys. J. Spec. Top. 2013. V. 222. N 10. P. 2399.

[25] Hoppensteadt F.C., Izhikevich E.M. // IEEE Trans. Neural Networks. 2000. V. 11. N 3. P. 734.

[26] Hoppensteadt F.C. // Scholarpedia. 2006. V. 1. N 11. P. 1599.

[27] Best R.E. Phase-Locked Loops: Design, Simulation, and Applications. 5th ed. New York: McGraw-Hill, 2003.

[28] Дмитриев А.С., Клещов А.В., Кузьмин Л.В. // Успехи современной радиоэлектроники. 2008. № 1. С. 46.

[29] Morgan D.K. // TI Appl. Rep. SCHA002A. 2003.

[30] Izhikevich E.M. Dynamical Systems in Neuroscience: The Geometry of Excitability and Bursting. 1st ed. Boston: The MIT Press, 2007.

2 Письма в ЖТФ, 2017, том 43, вып. 13 
[31] Jensen M., Yaari Y. // J. Neurophysiol. 1997. V. 77. P. 1224.

[32] Чернышев И.В., Мищенко М.А., Матросов В.В. Патент на полезную модель № 158122. Российская Федерация, 2015.

[33] Binczak S., Jacquir S., Bilbault J.-M. et al. // Neural Netw. 2006. V. 19. N 5. P. 684.

[34] Horch K., Dhillon G. // Neuroprosthetics: theory and practice / Ed. Horch K.W., Dhillon G.S. World Scientific, 2004. V. 2.

[35] Bonifazi P., Difato F., Massobrio P. et al. // Front. Neural Circuits. 2013. V. 7. N March. P. 40. 